\title{
Lysosomal enzymes in the macronucleus of Tetrahymena during its apoptosis-like degradation
}

\author{
$E \mathrm{Lu}^{1}$ and $\mathrm{J}$ Wolfe ${ }^{*, 1}$ \\ ${ }^{1}$ Biology Department, Wesleyan University, Middletown, CT 06459, USA \\ * Corresponding author: J Wolfe, Biology Department, Wesleyan University, \\ Middletown, CT 06459, USA. Tel: 860 685-3239; Fax: 860 685-3279; \\ E-mail: jwolfe@wesleyan.edu
}

Received 20.7.00; revised 6.10.00; accepted 2.11.00

Edited by L Schwartz

\begin{abstract}
A key characteristic of apoptosis is its regulated nuclear degradation. Apoptosis-like nuclear degradation also occurs in the ciliated unicellular organism, Tetrahymena thermophila. Chromatin of the macronucleus undergoes massive condensation, a process that can be blocked by caspase inhibitors. The nucleus becomes TUNEL-positive, and its DNA is cleaved into nucleosome-sized fragments. In a matter of hours the macronucleus is completely degraded, and disappears. The condensed nucleus sequesters acridine orange, which means that it might become an acidic compartment. We therefore asked whether lysosomal bodies fuse with the condensed macronucleus to form an autophagosome. We monitored acid phosphatase (AP) activity, which is associated with lysosomal bodies but is not found in normal nuclei. We find that after the macronucleus condenses AP activity is localized in cap-like structures at its cortex. Later, after the degrading macronucleus loses much of its DNA, acid phosphatase deposits appear deeper within the nucleus. We conclude that although macronuclear elimination is initiated by an apoptosis-like mechanism, its final degradation may be achieved through autophagosomy. Cell Death and Differentiation (2001) 8, 289-297.
\end{abstract}

Keywords: acidification; apoptosis; autophagosome; ciliate; lysosome; nucleus

Abbreviations: $P C D$, programmed cell death; $A O$, acridine orange; LB, Iysosomal bodies; DAPI, 4,6-diamidino-2-phenylindole; TUNEL, Tdt-mediated dUTP nick end-labeling

\section{Introduction}

Apoptosis, or programmed cell death (PCD), is essential for the development and the homeostatic maintenance of multicellular organisms. ${ }^{1,2}$ It is a morphologically distinct form of cell death that can result in the rapid removal of unwanted or potentially dangerous cells. Apoptotic cells become small and compact, and their nuclei also become condensed and inactivated. Indeed, nuclear condensation is one of the hallmarks of apoptosis, and is a phenomenon of special significance to the present study.

Ciliates, like Tetrahymena, are binuclear organisms with two distinct types of nuclei, macro- and micronuclei. The polyploid macronucleus is a site of synthesis of mRNA and it governs the somatic phenotype of the cell, while the transcriptionally inactive diploid micronucleus serves as the germ nucleus. During the course of sexual conjugation in Tetrahymena, the macronucleus is degraded. The micronucleus undergoes meiosis and gives rise to haploid pronuclei, which engage in fertilization. The zygote nucleus divides and differentiates into a new micro- and macronucleus, while the old macronucleus is eliminated. Degradation of the old macronucleus occurs by a process that shares similarities to apoptotic nuclear degradation in higher cells. ${ }^{3,4}$

Chromatin becomes highly condensed, and DNA is digested by a mechanism that results in oligonucleosomesized fragments. ${ }^{3,4}$ Chromatin condensation is preceded by DNA cleavage into very high molecular weight fragments. ${ }^{4}$ The process is under gene control, since it is blocked by inhibitors of protein and RNA synthesis. ${ }^{4}$ The condensed nucleus contains fragments of DNA as attested by positive TUNEL staining. ${ }^{4}$ Finally, caspase inhibitors block nuclear condensation. ${ }^{5}$ All of these characteristics relate macronuclear elimination in Tetrahymena to apoptosis in higher organisms. Therefore, the developmentally regulated elimination of the macronucleus during sexual conjugation in Tetrahymena thermophila represents a special case of apoptosis, one where the nucleus 'dies', but the cell remains alive.

During a study of living conjugants, it was observed that acridine orange (AO)-positive vesicles are preferentially localized in the posterior portion of the cell and become tightly clustered around the condensed macronucleus. ${ }^{6} \mathrm{AO}$ is modified by protons making it membrane impermeable. ${ }^{7}$ Since AO accumulates in membrane-bound acidic vesicles, positively staining structures are thought to be lysosomally derived bodies. ${ }^{8} \mathrm{AO}$ has been used to detect apoptotic cells in Drosophila embryos, ${ }^{9}$ suggesting that acidification may be associated with apoptosis in that system, as well.

By combining $\mathrm{AO}$ with Hoechst 33342, a vital stain which binds specifically with DNA, we were able to observe blue nuclei and orange lysosomal bodies (LBs) simultaneously in living cells. We discovered that after condensation the macronucleus does not stain blue like other nuclei, but appears yellow or orange. Nuclei of apoptotic avian chondrocytes also appear yellow or orange with this stain. ${ }^{6}$ After meiosis in Tetrahymena, degenerating haploid nuclei also become yellow and orange. ${ }^{10}$ Because this combination of dyes distinguishes apoptotic nuclei from normal nuclei in living cells, we refer to it as an 'apofluor'. 6 
Beyond the practical utility of 'apofluor' in identifying dying nuclei while cells still are alive, the data suggested that apoptotic nuclei were becoming acidic, like lysosomal bodies. A number of studies have suggested an important role for acidification in apoptosis. ${ }^{11-13}$ In this system, acidification of the macronucleus might be related to its apoptosis-like degradation.

Yet how could acidification of the macronucleus be accomplished? One possibility is that the envelope of an apoptotic nucleus becomes sealed and a proton pump is activated, increasing the intra-nuclear $\mathrm{pH}$. Alternatively, and more in keeping with our observations, the putative acidification of the condensed macronucleus is somehow related to the clustering of LBs around it. Lysosomal bodies might actively fuse together, then envelop and incorporate the macronucleus into an autophagosome.

If so, then we might expect lysosomal hydrolytic enzymes to become incorporated into the condensed macronucleus. To test this idea, we investigated whether acid phosphatase, a lysosomal enzyme,$^{14-16}$ is localized to the macronucleus during its apoptosis-like degeneration. In the course of this study we worked out a procedure for simultaneously visualizing nuclear structures by fluorescence microscopy and opaque products of acid phosphatase activity by bright field emission. This approach might be useful for studying nuclear elimination in other apoptotic systems as well.

Using this method, we find that acid phosphatase activity does not co-localize with normal nuclei, but does colocalize with the degenerating macronucleus. This observation supports the hypothesis that the apoptotic macronucleus is transformed into an autophagosome.

\section{Results}

A diagram summarizing the main features of nuclear changes during conjugation in Tetrahymena is shown in Figure 1. Of particular interest in the present study are stages k-p during which the chromatin of the old macronucleus becomes highly condensed, the condensed macronucleus moves, generally, to the posterior end of the cell, the DNA of the old macronucleus is digested, and the structure disappears.

Figure $2 \mathrm{a}-\mathrm{c}$ shows a set of DAPI stained conjugants to illustrate this process in actual cells. An arrow points to the regular, large macronucleus in Figure 2a; a single zygote micronucleus is also seen in these cells (comparable to Figure 1d). After the zygote nucleus divides twice mitotically, two developing macronuclei and micronuclei are produced (Figure $2 \mathrm{~b}$ ). At this stage (comparable to Figure $1 \mathrm{~m}$ ), the macronucleus is condensed (arrow) and has moved to the posterior end of the cell. In a few hours, the condensed macronucleus disappears, leaving two developing macronuclei and two micronuclei (Figure 2c). This stage is comparable to Figure $1 p$.

Visualization of living cells with vital apofluor reveals that lysosomal bodies (stained orange) and nuclei (stained blue) can be seen simultaneously (Figure 3a). We have observed that just prior to macronuclear condensation lysosomal bodies (LB's) tend to accumulate at the posterior end of the cell, and are often clustered near the macronucleus. ${ }^{6,10}$

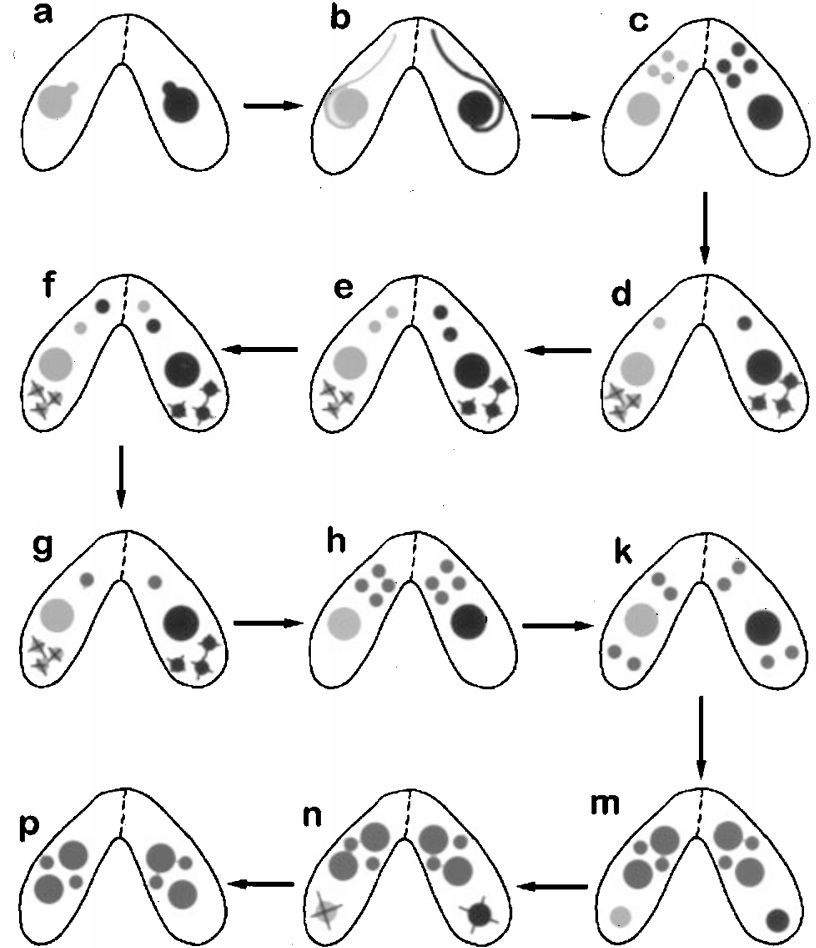

Figure 1 A diagram illustrating key stages in nuclear events during conjugation. The micronucleus moves away from its residence at the macronuclear envelope (a) and elongates to a length greater than that of the cell (b). After it contracts it undergoes meiosis to yield four haploid nuclei (c). Three haploid nuclei degenerate (d) while the remaining one divides mitotically to yield migrating and stationary pronuclei (e). After translocation (f) and nuclear fusion a zygote nucleus is formed $(\mathbf{g})$. The zygote nucleus divides twice mitotically $(\mathbf{h})$ to produce two developing macronuclei and two micronuclei (k), while the old macronucleus condenses and moves posteriorly $(\mathbf{m})$. The old macronucleus is degraded (n) and disappears (p)

After macronuclear condensation, the old macronucleus is stained yellow, a combination of blue and orange, suggesting that the nucleus has become acidified (Figure 3b).

When Hoechst 33342 was used together with Euchrysine, an acridine derivative which stains lysosomal bodies green, ${ }^{17}$ the pre-condensed old macronucleus, along with four post-zygotic nuclei, were stained blue, while LBs, which concentrated at the posterior end of each cell, were stained green (Figure 4a). This indicates that the two dyes retain their staining specificities when combined. In a later conjugant pair (Figure 4b), with its old macronucleus already condensed, the staining pattern was altered. The condensed macronucleus was differentially stained green, similar to LBs, rather than blue. This staining procedure yielded results similar to that of apofluor, albeit with different coloration, supporting the suggestion that the old macronucleus becomes acidified upon condensation.

Figure $5 \mathrm{a}$ shows the kinetics of pairing over a 24-h period. Cells begin to form pairs at about $1 \mathrm{~h}$ after mixing, and by $10 \mathrm{~h}$ they begin to come apart. It was previously established that, under these conditions, macronuclear degradation occurs predominantly between hours 8 and 

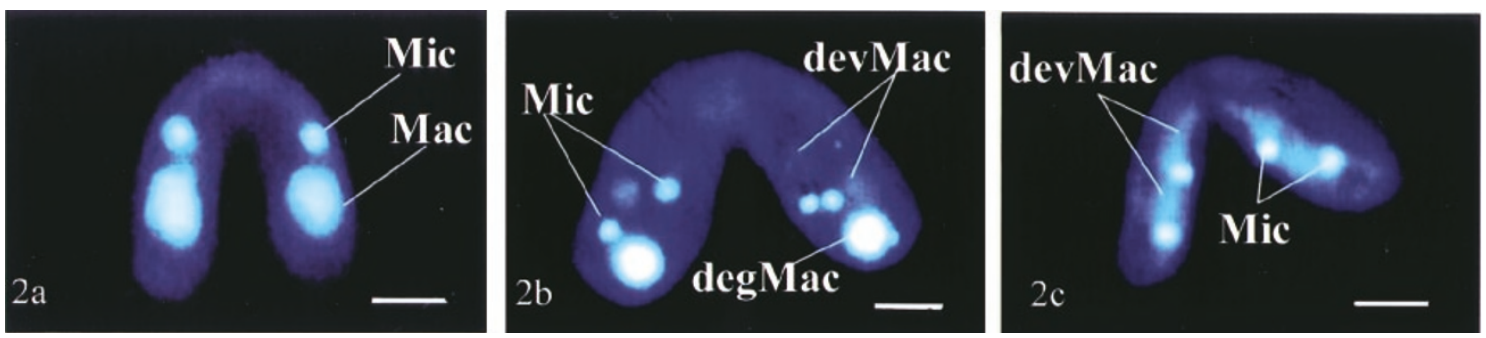

Figure 2 (a) DAPI stained fixed conjugants with a regular large macronucleus and a single zygote micronucleus. (b) DAPI stained conjugants with two developing macronuclei and micronuclei and a condensed macronucleus located at the posterior end of the cell. (c) Conjugant pairs stained with DAPI after macronuclei have been eliminated leaving two micronuclei and two developing macronuclei. Bar $=10 \mu \mathrm{m}$
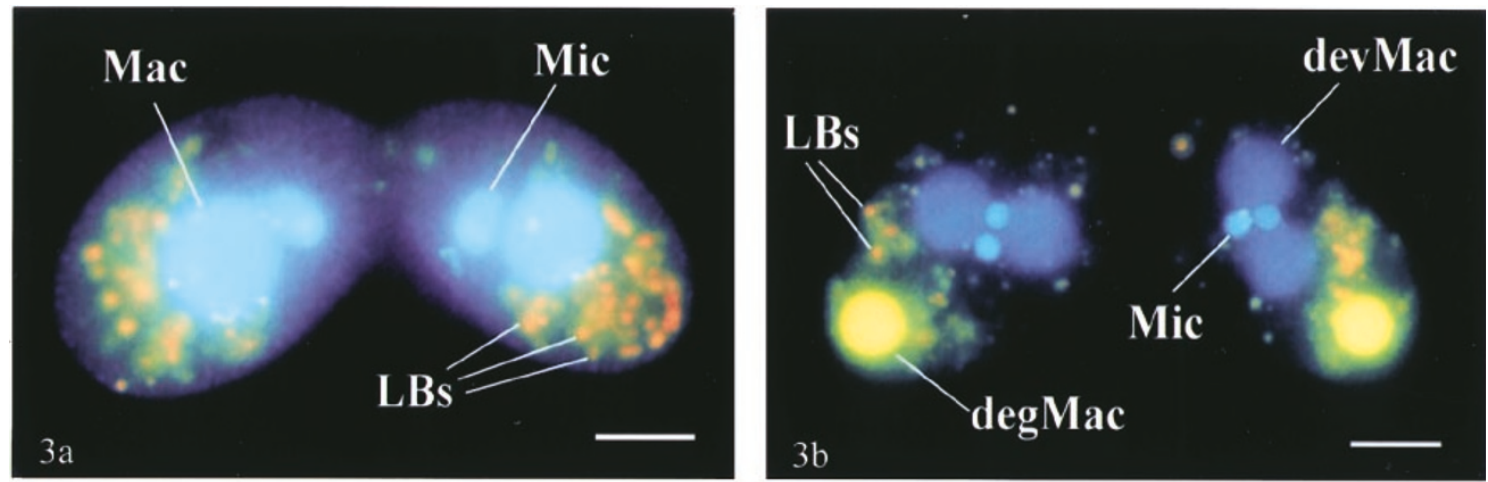

Figure 3 Anesthetized conjugant pairs stained with apofluor. (a) Micronucleus and macronucleus are blue, and lysosomal bodies are stained orange. (b) Micronuclei and developing macronuclei are blue (two each), but the condensed macronucleus is stained differentially yellow, a combination of Hoechst and Acridine Orange staining. Bar $=10 \mu \mathrm{m}$
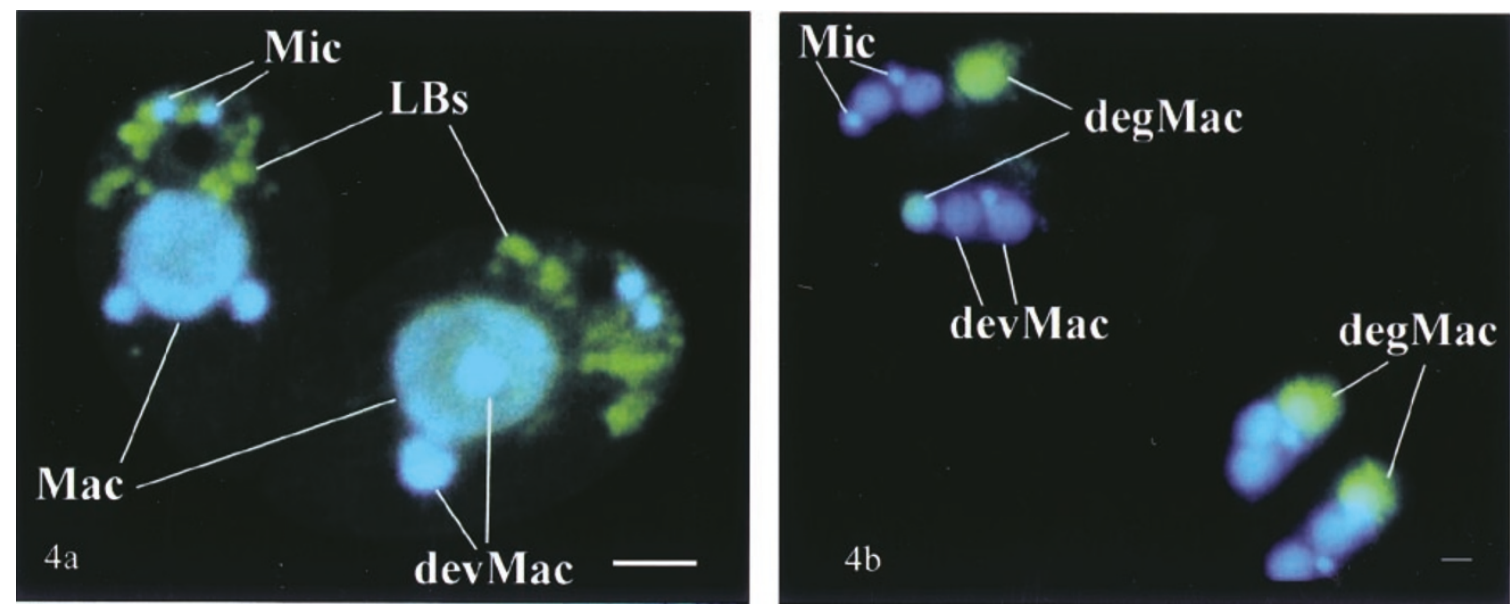

Figure 4 Anesthetized conjugant pairs stained with euchrysine. (a) Micronucleus and macronucleus are blue while lysosomal bodies are stained green. (b) Micronuclei and developing macronuclei are blue, but the condensed, degrading macronucleus is differentially stained green, indicating that the condensed macronucleus stains like lysosomes. Bar $=10 \mu \mathrm{m}$

16 after mixing mating types. ${ }^{4}$ We measured acid phosphatase activity during a 24-h period, using a colorimetric assay, ${ }^{18}$ but found that the level was invariant (Figure 5b). Though data from a single experiment are shown, the experiment was repeated and the results were confirmed. We conclude that there is no significant change in the amount of acid phosphatase activity during conjugation associated with macronuclear degradation.

We next conducted experiments to localize acid phosphatase. The classic Gömöri method gave high contrast results, and DAPI could be successfully used to identify nuclei. However in some single cells, the nucleus 

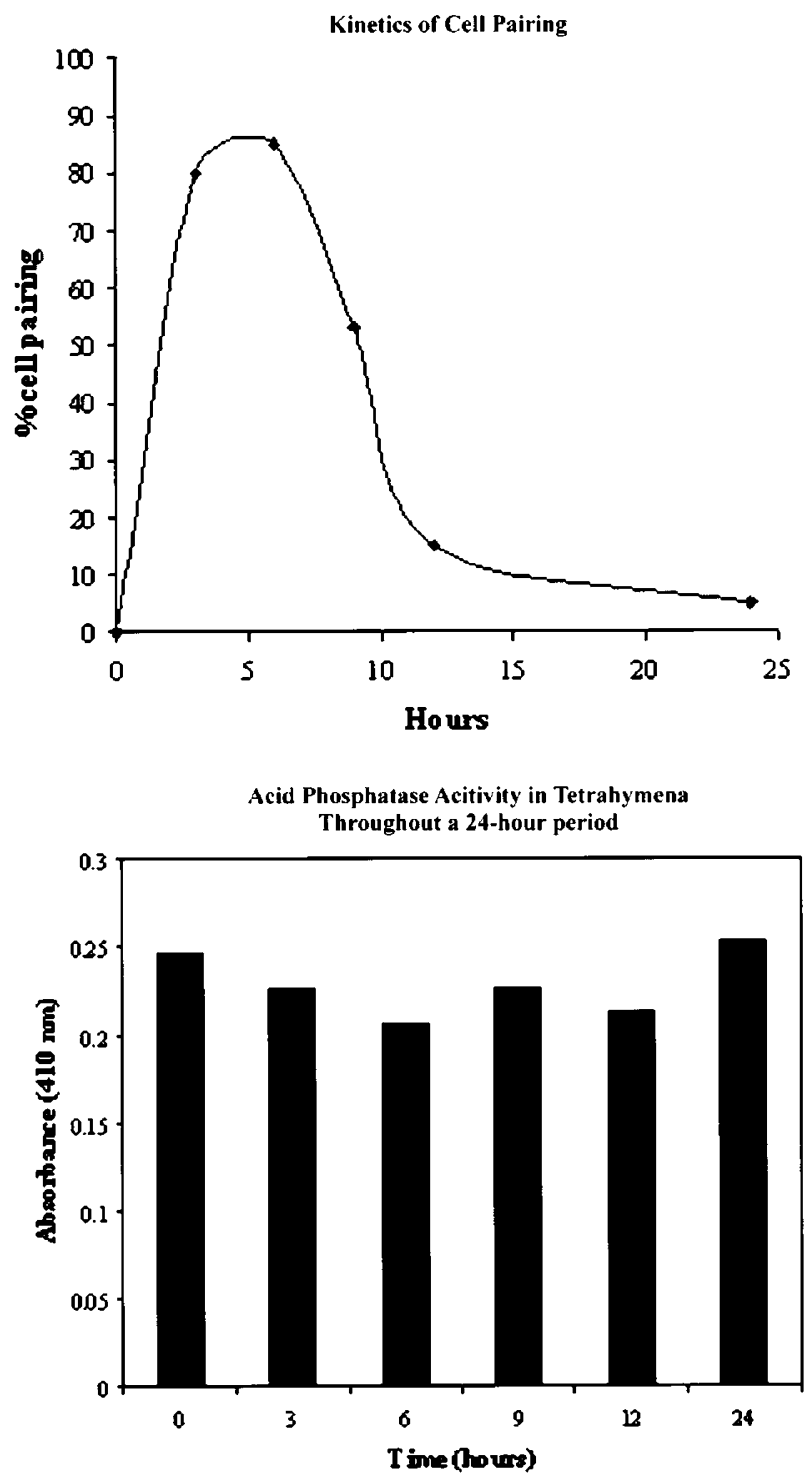

Figure 5 (a) Kinetics of cell pairing and dissociation. Cells begin to pair a about $1 \mathrm{~h}$ after mixing mating types, and start to dissociate after $10 \mathrm{~h}$. The macronucleus continues to be degraded in the post-paired single cells. (b) Acid phosphatase activity during conjugation, measured colorimetrically, for a 24-h period. Activity remains constant during this period indicating that there is no significant change in lysosomal content during conjugation. Bar $=10 \mu \mathrm{m}$

also stained positively for acid phosphatase, an artifact resulting from diffusion of the initial reaction product and entrapment in chromatin, before being deposited as a lead precipitate. $^{19}$

Despite a series of experiments in which we varied substrate concentrations and incubation times, it was not possible, in our hands, to eliminate this artifact. Since the test of our hypothesis depended on an ability to visualize acid phosphatase product newly associated with the condensed old macronucleus, the Gömöri method was precluded from use.

With the Azo Dye method, on the other hand, nuclei were not detectable by DAPI, whether used before or after the procedure. This meant that it was not possible to see apoptotic nuclei and acid phosphatase product simultaneously. Apparently, there was some interaction between DAPI and the Azo Dye method that made it impossible to visualize DAPI. This also proved true for other fluorescent stains as well, including ethidium bromide, propidium iodide acridine orange, Hoechst 33342, and Euchrysine. (Acridine orange can be used with fixed cells to stain nuclei). ${ }^{20}$ Apparently, the Azo Dye procedure did not just interfere with DAPI stain, but with fluorescent DNA stains in general.

However if the Azo Dye method is used after cells are fixed in $20 \%$ formaldehyde neutralized in $0.01 \mathrm{M}$ phosphate buffer, $\mathrm{pH} 7.2$, the same fixative we use for assaying cell pairing, rather than the $2 \%$ glutaraldehyde called for in the published procedure, double staining can be achieved. Using fluorescence together with dim brightfield illumination, we find it possible to visualize both the acid phosphatase product and DAPI-stained nuclei simultaneously (Figure 6a). This allowed us to test our hypothesis.

Figure $6 \mathrm{~b}$ shows a conjugant pair at $9 \mathrm{~h}$ after mixing mating types stained by the Azo Dye method and viewed by brightfield microscopy. Acid phosphatase product, in addition to being localized to particulate bodies in the posterior end, is also seen as an uneven ring of material in the posterior end of the cell. The same cell pair is viewed in Figure $6 \mathrm{c}$ by fluorescence microscopy. It is apparent that the uneven circle of black material in Figure $6 \mathrm{~b}$ corresponds to small cap-like deposits localized to the cortical zone of the condensed degenerating macronucleus. In Figure 6d, a conjugant pair is observed simultaneously with brightfield and fluorescence microscopy. Acid phosphatase deposits completely encircle the condensed nuclei.

It is clear that an intimate association develops between lysosomes and the degrading macronucleus, but it is not possible to tell whether the lysosomal structures are pressed against the macronucleus, or whether they have actually enclosed it in an autophagosome. We therefore examined macronuclear degradation at later stages. As chromatin is digested more and more lysosomal enzyme might enter into the interior of the macronucleus. One might also expect to see an inverse relationship between the strength of the DAPI stain and the extent to which acid phosphatase has moved to the interior of the structure. This, indeed, seems to be the case.

Figure $6 \mathrm{e}$ shows macronuclei in conjugants at $14 \mathrm{~h}$ after mixing mating types. In the upper cell acid phosphatase stains strongly at the condensed macronucleus and black deposits clearly co-localize with the fluorescence. This indicates that lysosomal enzyme enters into the interior of the macronucleus as digestion proceeds. Interestingly, in the bottom cell, even though the macronucleus can no longer be detected by DAPI staining, acid phosphatase deposits are located exactly where the dying macronucleus would have been, based on its position in the upper cell. This indicates that even after DNA has been digested to the point where it is no longer visible by DAPI-staining a residual entity can be detected by virtue of its acid phosphatase content.

Figure $6 f$ shows an even later stage; a single cell that had separated from a pair towards the end of conjugation. 

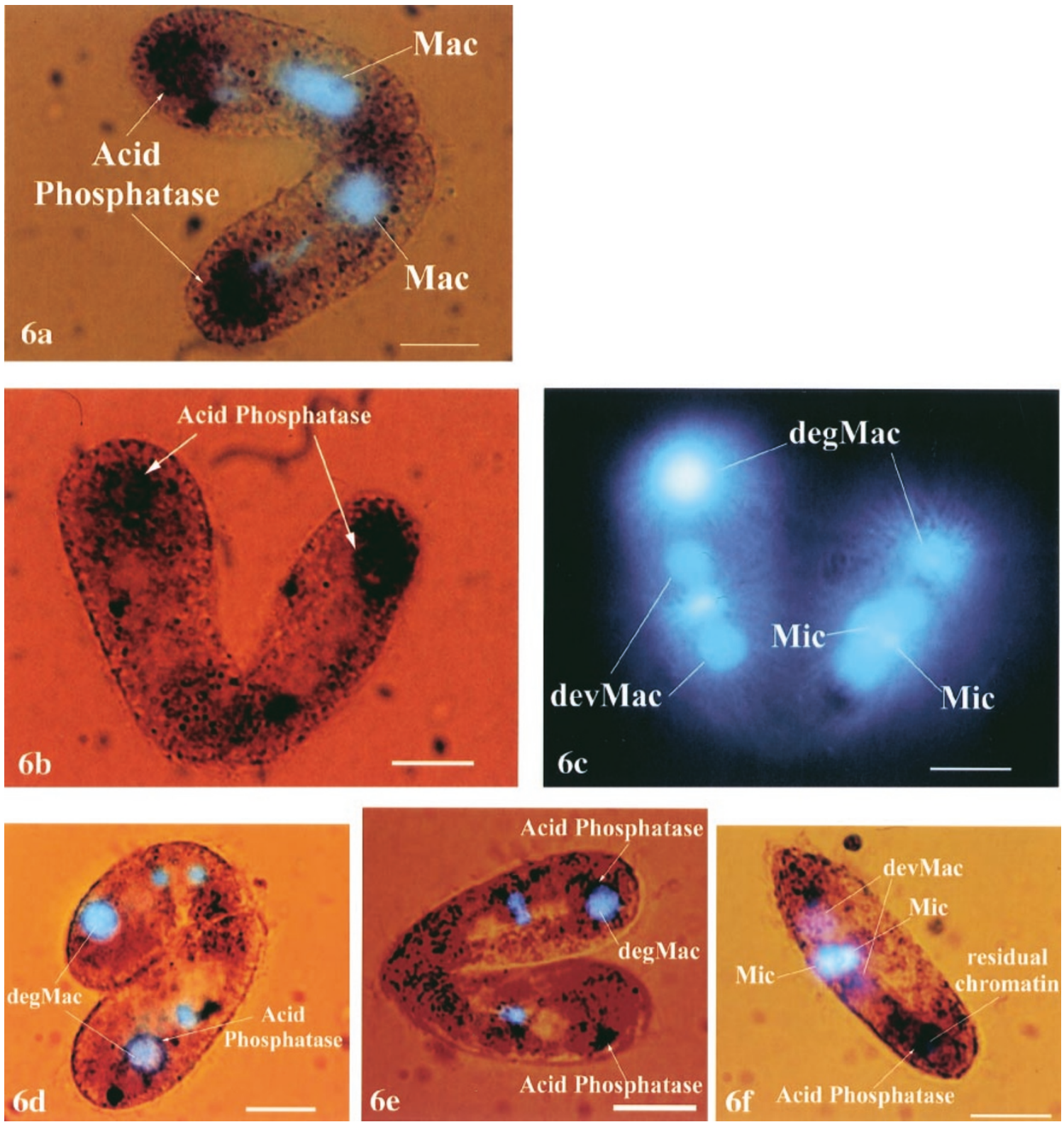

Figure 6 (a) Localization of acid phosphatase activity using the modified Azo Dye method, showing simultaneous DAPI staining of the macronucleus with fluorescence microscopy, and dark deposits corresponding to lysosomal bodies, seen with bright field microscopy. The macronucleus is free of acid phosphatase product. (b) A conjugant pair viewed by brightfield microscopy, after staining for acid phosphatase by the Azo Dye method. Here, in addition to aggregates of granules, concentrations of dark deposits are seen in a circle at the posterior end of the cell, as if at the cortex of a condensed macronucleus. (c) The same pair viewed by fluorescence microscopy showing that the dark deposits in $\mathbf{b}$ are localized to condensed macronuclei, but not to developing macronuclei or micronuclei. (d) A pair of conjugant cells at $9 \mathrm{~h}$ after mixing, stained by Azo Dye and DAPI, and viewed simultaneously with brightfield and fluorescence microscopy. In the lower cell the condensed macronucleus is ringed with black deposits at its cortex. (e) A pair of 14-h conjugants. Dark deposits, indicative of AP activity, fill the condensed macronucleus of the upper cell. In the lower cell AP deposits occupy a site where the condensed macronucleus should be, but DAPI positive material is not seen, possibly because the DNA has already been digested. (f) A single cell, at $14 \mathrm{~h}$ after mixing mating types, resulting from pair separation. A slight amount of DAP positive material is associated with a large deposit from AP activity located in the posterior of the cell. Bar $=10 \mu \mathrm{m}$

DAPI-stained newly developed macronuclei and micronuclei are in the middle of the cell. No discrete condensed macronucleus is visible with DAPI, and only a tiny amount of fluorescence was detected, indicating a small amount of DNA remaining. Again, acid phosphatase product is seen, revealing a residual entity that is clearly an old macronucleus in a late stage of degradation.

\section{Discussion}

These data show that the condensed, degenerating macronucleus of Tetrahymena, in addition to becoming acidic, as inferred from apofluor staining, also comes to contain acid phosphatase. Since acid phosphatase is a lysosomal enzyme, and its activity can be used to cytochemically 
identify lysosomes, we can infer that lysosomes combine their contents with the macronucleus. In so doing, they form a nuclear autophagosome.

Autophagy, or self-digestion, is a general means for the intracellular disposal of obsolete proteins or organelles. Two different pathways for autophagosome formation have been described.

In one, membranous outpocketings from the endoplasmic reticulum surround components such as aged mitochondria and sequester them from the remaining cytoplasm. Secondarily, lysosomes fuse with the doublemembranes enclosing structure, and when the inner membrane dissolves, an autophagosome is formed. Degradation of the engulfed organelles ensues. ${ }^{21,22}$

In the second model, which does not involve the endoplasmic reticulum, primary lysosomes flatten and fuse with one another and spread directly as a double membrane structure enclosing cytoplasmic areas or organelles to be degraded. ${ }^{23,24}$ When the inner membrane of the flattened lamella disintegrates, it leaves an autophagosome enclosing the component(s) to be digested. ${ }^{25,26}$

In yeast, autophagy occurs by a modification of the lysosomal wrapping pathway. ${ }^{27}$ In that system the large acidic vacuole contains a wide variety of hydrolases and is considered to be homologous to lysosomes in animal cells. ${ }^{28,29}$ The process of autophagy involves formation of a double-membrane structure derived from the vacuole, which wraps around cytoplasmic components or organelles. This is followed by disintegration of the inner membrane. Once this occurs, an autophagosome is formed and hydrolytic enzymes inside the vacuole digest the enclosed cellular material. ${ }^{30}$

From the results presented here, it appears that autophagy in Tetrahymena also occurs by the direct lysosomal pathway. Just prior to macronuclear condensation, acid phosphatase-containing bodies corresponding to acridine orange positive vesicles, become preferentially localized to the posterior end of the cell. They then aggregate around the nucleus as it condenses. The condensed nucleus becomes acridine orange-positive, and is differentially stained by apofluor. At this stage acid phosphatase-containing 'caps' appear at the nuclear envelope. This material increases until it completely encircles the macronucleus. At a later time, when macronuclear degradation is at a more advanced stage, acid phosphatase becomes localized deeper inside the nucleus.

The co-localization of acid phosphatase with DAPIpositive material supports the hypothesis that lysosomal material and chromatin become enclosed in a common compartment. That fulfills the criterion for nuclear autophagosomy. Further, our findings are consistent with electron microscopic observations which showed that vesicles and a flattened lamella appear at the cortex of the condensed macronucleus. ${ }^{31}$ On the basis of the cytochemistry reported here, we can now safely assume that those vesicles correspond to lysosomal bodies, that the lamella is derived from their fusion, and that its presence at the cortex of the macronucleus represents autophagosomy in process. The sequence of events in this proposal most closely corresponds to the lysosomal-wrapping model, ${ }^{23,24}$ and is illustrated in Figure 7.

Lysosomes are increasingly thought to play an important part the clearing of apoptotic bodies in multicellular organisms. $^{32}$ Neighboring cells phagocytose cell fragments, some of which include nuclei, or nuclear pieces, that break off from apoptotic cells. ${ }^{33}$ Thymic nurse cells enclose apoptotic thymocytes within specialized intracellular vacuoles during $\mathrm{T}$ cell development. ${ }^{34}$ In $C$. elegans the significance of clearing apoptotic bodies by phagocytosis has been studied through mutational analysis. ${ }^{35}$ It has now been reported, for both mice and $C$. elegans, that phagocytosis is necessary for DNA digestion in mutant apoptotic cells that lack the functional capacity to autonomously conduct nuclear degradation. ${ }^{36,37}$ In these cases it is the lysosomes of the phagocytes that are responsible for the degradation. ${ }^{32}$ Nuclear autophagy during macronuclear elimination in Tetrahymena probably represents the equivalent process, though performed intracellularly.

Based on these studies we propose a two-stage process, apoptotic induction followed by autophagic completion, for the elimination of the old macronucleus in Tetrahymena. Apoptotic induction is evidenced by TUNEL staining, ${ }^{4}$ digestion of DNA into nucleosome-sized multiples, ${ }^{3,4}$ an elevation of caspase activity during macronuclear elimination and the blockage of macronuclear condensation by inhibitors of caspase activity. ${ }^{5,38}$ Caspase activity may be related to the initial cleavage of DNA into large pieces, since such cleavage may initiate chromatin condensation, ${ }^{39-41}$ and inhibition of DNase activity by aurin prevents macronuclear condensation. ${ }^{4}$ The digestion of DNA into nucleosome sized fragments may also be controlled by caspases, as in mouse cells where an inhibitor of DNase is cleaved by a caspase, thus liberating its activity. ${ }^{42}$

The second stage, autophagy, is evidenced by the apparent acidification of the condensed macronucleus, ${ }^{6}$ the localization of acid phosphatase to the macronucleus as it degrades, and the electron microscopic demonstration of lamellar vesicles enclosing the condensed nuclear structure. $^{31}$

It is an extraordinary feature of ciliates, resulting from its binuclear condition, that a nucleus can be eliminated even while the cell continues to live. This feature may make such cells particularly useful for analysis of the specific regulation of nuclear death, apart from the degradation of the rest of the cell. It also raises the interesting question about how nuclei in a common cytoplasm are distinguished from one another. Postzygotic nuclear products in a common cytoplasm differentiate into macronuclei or micronuclei ${ }^{43,44}$, and in the same cytoplasm, some nuclei disintegrate and disappear while others remain. It will be fascinating to find out how certain specific nuclei are selectively designated for differentiation or for elimination.

Some have proposed that apoptosis developed in multicellular organisms as a way to eliminate unwanted or malfunctioning cells, e.g. ${ }^{45}$ However, our studies on macronuclear elimination in Tetrahymena suggest that the 

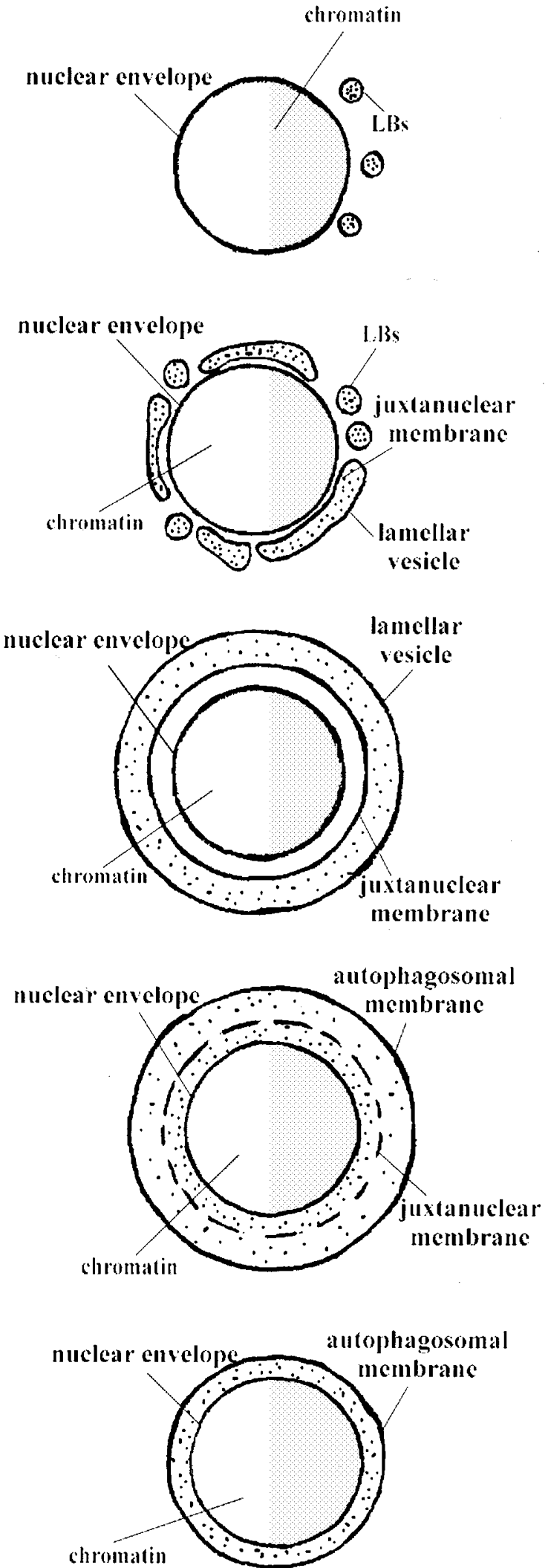

Figure 7 A diagram illustrating a possible sequence of events leading to the formation of an autophagosome in which the condensed macronucleus would come to possess AP activity machinery for apoptosis may have originated in unicellular organisms. The occurrence of some form of regulated cell death in other unicellular organisms and protistans, ${ }^{46-50}$ also supports this view. Perhaps mechanisms developed early in evolution were later adapted by multicellular organisms for the selective elimination of particular cells. ${ }^{51}$

\section{Materials and Methods}

\section{Cell growth}

Cells used in this study were mating types 2086 and 428 of Tetrahymena thermophila, developed by Peter Bruns at Cornell University. Concentrated $(10 \times)$ medium, stored at $-20^{\circ} \mathrm{C}$ in capped bottles, was prepared as 10\% proteose peptone (Difco), 1\% yeast extract, $1 \%$ glucose, and $0.001 \% \mathrm{Fe}^{2+}$ EDTA. The concentrate was thawed and diluted 10-fold for growth medium, as needed, and autoclaved.

Stock cultures were grown with $2 \mathrm{ml}$ medium in a $5-\mathrm{ml}$ disposable culture tube and were subcultured every 2 weeks or so. For experiments, larger cultures were prepared, e.g. $50 \mathrm{ml}$ of medium in 250-ml capped bottles. Cells were grown for 2-3 days, without agitation, to early stationary phase $\left(\sim 2.5 \times 10^{5}\right.$ cells $\left./ \mathrm{ml}\right)$ at $30^{\circ} \mathrm{C}$.

\section{Conjugation}

To prepare for conjugation, cells were washed by low speed centrifugation $(800 \times g$ in an IEC-PRG refrigerated centrifuge) twice

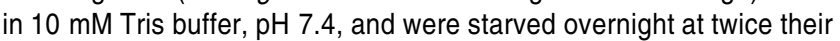
cell density. Equal volumes of two complementary mating types were mixed in sterile containers with a surface area to volume ratio of $20: 1$ and were incubated at $30^{\circ} \mathrm{C}$. Samples of $0.1-0.2-\mathrm{ml}$ aliquots were transferred to microfuge tubes at different time points, and were fixed with an equal volume of $20 \%$ formaldehyde neutralized with $0.01 \mathrm{M}$ phosphate buffer, $\mathrm{pH} 7.2$.

Cell pairing was analyzed by phase contrast microscopy using a $20 \times$ objective. A microscope slide was scanned from ones side of a coverslip to the other, back and forth until 200 cells were scored. The percentage of pairing was calculated using the formula: number of cells in pairs $\times 100$ divided by the total number of cells as single cells and cells in pairs. Typically, pairing was first observed at $1 \mathrm{~h}$ after mixing and cell pairing reached $70-80 \%$ by $4 \mathrm{~h}$.

\section{Acid phosphatase colorimetric assay}

Acid phosphatase activity was assayed colorimetrically, ${ }^{18}$ using a kit obtained from Sigma. Cell samples $(10 \mathrm{ml})$ were taken at various times during conjugation in a 24-h period. After centrifugation at $800 \times g$ for $5 \mathrm{~min}$ at $4^{\circ} \mathrm{C}$ r.p.m., pellets were lysed by adding $2 \mathrm{ml} 0.3 \mathrm{M} \mathrm{KCl}+0.1 \%$ Triton X-100. The lysate was centrifuged at $1800 \times g$ to remove cell debris. The resulting supernatant was transferred to 1.5-ml Eppendorf tubes and stored at $-20^{\circ} \mathrm{C}$ until use. Absorbance was measured with a Spectronic 20 spectrophotometer at $410 \mathrm{~nm}$.

\section{Cytochemical localization of acid phosphatase}

Method I Following the procedure of Gömöri, ${ }^{52}$ cells were fixed on ice for a minimum of $30 \mathrm{~min}$ in $2 \%$ glutaraldehyde, freshly prepared in $0.05 \mathrm{M}$ phosphate buffer, $\mathrm{pH}$ 7.4. After washing in $10 \mathrm{ml}$ of $0.05 \mathrm{M}$ sodium acetate buffer, $\mathrm{pH} 5.0$, cells were resuspended in a $5-\mathrm{ml}$ reaction solution with a final concentration of $10 \mathrm{mM} \beta$ - 
glycerophosphate and $3 \mathrm{mM}$ lead nitrate. At the end of a 30 - or $45-$ min incubation period at $37^{\circ} \mathrm{C}$, cells were washed in $5 \mathrm{ml}$ of $10 \mathrm{mM}$ Tris buffer, $\mathrm{pH} 7.4$, three times to terminate the reaction. A $1-\mathrm{m}$ aliquot was treated with $1 \mathrm{ml}$ of $1 \%$ sodium sulfide for $5 \mathrm{~min}$. The sample was washed in $10 \mathrm{mM}$ Tris buffer, $\mathrm{pH} 7.4$ and resuspended in distilled water.

Method II Using the AzoDye procedure of Anderson, ${ }^{53} 5-\mathrm{ml}$ samples of cells were fixed at various times after mixing mating types in an equal volume of $2 \%$ glutaraldehyde (in later experiments, $20 \%$ formaldehyde) in $0.05 \mathrm{M}$ phosphate buffer, $\mathrm{pH} 7.4$ on ice for a minimum of $30 \mathrm{~min}$. They were washed with $2.5 \mathrm{M}$ sodium acetate buffer, $\mathrm{pH} 5.2$, three times, to completely remove the fixative. Pellets were resuspended and incubated in $1 \mathrm{~m}$ of staining solution for $30 \mathrm{~min}$ at $37^{\circ} \mathrm{C}$. Staining solution $(50 \mathrm{ml})$ was prepared with $46.0 \mathrm{ml}$ of water at $37^{\circ} \mathrm{C}, 2.0 \mathrm{ml} 2.5 \mathrm{M}$ acetate buffer, $\mathrm{pH} 5.2,2.0 \mathrm{ml}$ of naphthol AS-BI phosphoric acid solution, and $15.0 \mathrm{mg}$ of Fast Garnet GBC salt, and was filtered. Two different control solutions were used. One consisted of $48.0 \mathrm{ml}$ of water, $2.0 \mathrm{ml} 2.5 \mathrm{M}$ acetate buffer, $\mathrm{pH} 5.2$, and $15.0 \mathrm{mg}$ of Fast Garnet GBC salt. The other lacked Fast Garnet. Staining solution was prepared freshly for each experiment and was brought to room temperature prior to incubation.

To terminate the reaction, samples were washed with $10 \mathrm{mM}$ Tris buffer, $\mathrm{pH} 7.4$, three times, and then resuspended in distilled water.

\section{Cytological analyses}

DAPI staining Nuclear morphology was observed by staining fixed cells with the fluorescent dye, 4,6-diamidino-2-phenylindole (DAPI). Two hundred-microliter samples were withdrawn at appropriate times and fixative was removed by centrifugation in a microfuge for $30 \mathrm{~s}$. Cells were resuspended in $200 \mu 10 \mathrm{mM}$ Tris and stained with $10 \mu \mathrm{l}$ of $10 \mu \mathrm{g} / \mathrm{ml}$ DAPI. After $5 \mathrm{~min}$ they were centrifuged again, and resuspended in $200 \mu \mathrm{l} \mathrm{NPG}(25 \%$ glycerol and $1 \% \mathrm{~N}$-propylgallate in $0.01 \mathrm{M}$ phosphate buffer at $\mathrm{pH} 7.0$ ) to retard photo-bleaching. ${ }^{54}$ Cells were observed with a fluorescence microscope using the filter for blue light.

Apofluor staining Macronuclear condensation and lysosomal activity were observed in living cells by staining with apofluor, prepared with two parts of $100 \mathrm{ug} / \mathrm{ml}$ acridine orange and one part of $1 \mathrm{mg} / \mathrm{ml}$ Hoechst $33342 .{ }^{6}$ One-ml sample of cells were taken at various times after mixing mating types, and transferred to $15-\mathrm{ml}$ conical centrifuge tubes, to which $10 \mu \mathrm{l}$ of apofluor was added. After $10 \mathrm{~min}$ of incubation with the vital stain, the cells were immobilized by anesthetization. Cells were observed with a fluorescence microscope using the filter for blue light.

Euchrysine staining Lysosomal activity was observed in living cells by staining the cells with euchrysine. One-ml samples of cells were taken at desired times and stained with $5 \mu \mathrm{l}$ of $1 \mathrm{mg} / \mathrm{ml}$ euchrysine for $10 \mathrm{~min}$ followed by immobilization of the cells by anesthetization. Stained cells were observed under a fluorescence microscope with a filter for green light.

\section{Anesthetization of living cells}

Cells were stained and immobilized simultaneously, as reported previously. ${ }^{10}$ In brief, one $\mathrm{ml}$ of cells was incubated with vital fluorescent stains for $10 \mathrm{~min}$ in a $15-\mathrm{ml}$ centrifuge tube. These cells were then centrifuged for $30 \mathrm{~s}$ in an IEC clinical centrifuge, and $900 \mu \mathrm{l}$ of supernatant removed. The pellet was resuspended in the remaining $100 \mu \mathrm{l}$ of fluid. While vortexing gently, $10 \mu \mathrm{l}$ of $4 \mathrm{mM}$ dibucaine (City Chemical Corp., NY, USA) in $0.01 \mathrm{M}$ Tris, pH 7.4 was added. The tubes were maintained at room temperature without agitation for exactly $85 \mathrm{~s}$ (after about $6 \mathrm{~h}$ of conjugation a $90-100-\mathrm{s}$ wait is necessary), and $9 \mathrm{ml}$ of $0.01 \mathrm{M}$ Tris ( $\mathrm{pH} 7.4$ ) with $0.01 \mathrm{M}$ sucrose was added. The cells were centrifuged for $30 \mathrm{~s}$, after which the supernatant was discarded, removing both the dibucaine and excess stain, and $1 \mathrm{ml}$ of fresh Tris/sucrose solution was added. Under these conditions, cells typically remained immobilized for about $30 \mathrm{~min}$.

\section{Microscopy and image analysis}

Observations were made with a Zeiss Axioplan microscope, using a $100 \times$ oil-immersion objective. Exposure time was either 8 or $15 \mathrm{~s}, 1 / 4$ of that prescribed by the automatic camera. Reciprocity was maintained at 5. Images were recorded using Fujichrome Sensia II 200 ASA slide film, transferred to a computer via a Polaroid SprintScan 35 Plus film scanner, and printed with a Kodak DS 8650 PS Color Printer, using Adobe Photoshop 5.0 to label and adjust the size and position of the images.

\section{Reagents}

Unless otherwise specified, all reagents were obtained from Sigma.

\section{Acknowledgements}

This work was supported by NIH grant \# GM18051, and with funds provided by Wesleyan University. We are grateful for the help of Marie K Santos, Dan Ballon and Chutima Talchoi, who took an active interest in every facet of the project.

\section{References}

1. Rich T, Watson C and Wyllie A (1999) Apoptosis: the germs of death. Nature Cell Biol. 1: E69-E71

2. Vaux D and Korsmeyer S (1999) Cell death in development. Cell 96: 245-254

3. Davis MC, Ward JG, Herrick G and Allis CD (1992) Programmed nuclear death: Apoptotic-like degradation of specific nuclei in conjugating Tetrahymena. Dev. Biol. 154: 419-432

4. Mpoke S and Wolfe J (1996) DNA digestion and chromatin condensation during nuclear death in Tetrahymena. Exp. Cell Res. 225: 357-365

5. Ejercito Rand Wolfe J (1998) Caspases and nuclear death in Tetrahymena. Mol. Biol. Cell 9S: 245a

6. Mpoke S and Wolfe J (1997) Differential staining of apoptotic nuclei in living cells: Application to macronuclear elimination in Tetrahymena. J. Histochem. Cytochem. 45: 675-683

7. Anderson Rand Orci L (1988) A view of acidic intracellular compartments. J. Cell Biol. 106

8. Zelenin A (1966) Fluorescence microscopy of lysosomes and related structures in living cells. Nature 212: $425-426$

9. Steller H (1995) Mechanisms and genes of cellular suicide. Science 267: 14451449

10. Santos M, Lu E and Wolfe J (2000) Nuclear death in Tetrahymena: The case of the haploid nuclei. J. Euk. Microbiol. 47: 493-498

11. Fisher D (1994) Apoptosis in cancer therapy: crossing the threshold. Cell 78 : $539-542$

12. Gottlieb RA, Giesing HA, Zhu JY, Engler RL and Babior BM (1995) Cell acidification in apoptosis: Granulocyte colony-stimulating factor delays programed cell death in neutrophils by up-regulating the vacuolar $\mathrm{H}+-$-ATPase. Proc. Natl. Acad. Sci. USA 92: 5965-5968 
13. Monney L, Olivier R, Otter I, Jansen B, Poirier G and Borner C (1998) Role of an acidic compartment in tumor-necrosis-factor- $\alpha$-induced production of ceramide, activation of caspase-3 and apoptosis. Eur. J. Biochem. 251: 295-303

14. Bainton D (1981) The discovery of lysosomes. J. Cell Biol. 91: 66s - 76s

15. Holtzman E (1989) Lysosomes. (New York: Plenum)

16. Kornfeld S and Mellman I (1989) The biogenesis of lysosomes. Annu. Rev. Cell Biol. 5: 483-525

17. Allison A and Young $M$ (1964) Uptake of dyes and drugs by living cells in culture. Life Sci. 74: 1407-1414

18. Lau K, Farley J and Baylink D (1985) Phosphotyrosyl-specific protein phosphatase activity of a bovine skeletal acid phosphatase isoenzyme. Comparison with the phosphotyrosyl protein phosphatase activity of skeletal alkaline phosphatase. J. Biol. Chem. 260: 4653-4660

19. Barka T and Anderson P (1963) Histochemistry: Theory, Practice, and Bibliography. (New York: Harper Row)

20. Darzynkiewicz Z (1990) Simultaneous analysis of cellular RNA and DNA content. In: Methods in Cell Biology. Vol. 41, Darzynkiewicz Z, Robinson J, Crissman H, eds (San Diego: Academic Press) pp. 401-420

21. Dunn WJ (1994) Autophagy and related mechanisms of lysosome-mediated protein degradation. Trends Cell Biol. 4: 139-143

22. Yokota S, Himeno M, Roth J, Brada D and Kato K (1993) Formation of autophagosomes during degradation of excess peroxisomes induced by di-(2ethylhexyl)-phthalate treatment. II. Immunocytochemical analysis of early and late autophagosomes. Eur. J. Cell Biol. 62: 372-383

23. Mayahara H (1972) Ultracytochemistry of mouse subcutaneous histiocytes. 2. Mechanism of autophagolysosome formation. J. Kansai Med. School Suppl:98 109

24. Mayahara H and Ogawa K (1972) Lysosomal wrapping mechanism observed in the autophagolysosome formation. In: Histochemistry and Cytochemistry, Takeuchi T, Ogawa K, Fujita S, eds (Kyoto: Japan Soc. Histochem. Cytochem.) pp. $29-30$

25. Ogawa K (1981) Lysosomal wrapping mechanism (LWM) during autophagy. Acta Histochem. Cytochem. 14: 362-365

26. Sakai M and Ogawa K (1984) Relationship between lysosomal wrapping mechanism (LWM) and cytoskeletal elements during autophagolysosome formation. Acta Histochem. Cytochem. 17: 1-13

27. Kim J and Klionsky D (2000) Autophagy, cytoplasm-to-vacuole targeting pathway, and pexophagy in yeast and mammalian cells. Annu. Rev. Biochem. 69: $303-342$

28. Matile P and Wiemken A (1967) The vacuole as the lysosome of the yeast cell. Arch. Microbiol. 56: 148-155

29. Meyer $J$ and Matile $P$ (1975) Subcellular distribution of yeast invertase isoenzymes. Arch. Microbiol. 103: 51-55

30. Baba M, Takeshige K, Baba N and Ohsumi Y (1994) Ultrastructural analysis of the autophagic process in yeast: detection of autophagosomes and their characterization. J. Cell Biol. 124: 903-913

31. Weiske-Benner A and Eckert WA (1987) Differentiation of nuclear structure during the sexual cycle in Tetrahymena thermophila. II. Degeneration and autolysis of macro- and micronuclei. Differentiation 34:1-12

32. Ferri Kand Kroemer G (2000) Control of apoptotic DNA degradation. Nature Cell Biol. 2: E63-E64

33. Fadok V and Henson P (1998) Apoptosis: getting rid of the bodies. Curr. Biol. 8 : R693-R695

34. Samms M, Philp D, Emanus F, Osuji O, Pezzano M and Guyden J (1999) Lysosomal-mediated degradation of apoptotic thymocytes within thymic nurse cells. Cell Immunol. 197: 108-115
35. Wu $Y$ and Horvitz $H$ (1998) The $C$. elegans cell corpse engulfment gene ced-7 encodes a protein similar to $A B C$ transporters. Cell 93: 951-960

36. Wu Y-C, Stanfield G and Horvitz H (2000) NUC-1, a Caenorhabditis elegans DNase II homolog, functions in an intermediate step of DNA degradation during apoptosis. Genes Dev. 14: 536-548

37. Mcllroy D, TanakM, Sakahira H, Fukuyam H, Suzuki M, YamamuraK, Ohsawa Y, Uchiyama $Y$ and Nagata S (2000) An auxiliary mode of apoptotic DNA fragmentation provided by phagocytes. Genes Dev. 14: 549-558

38. Ejercito R (1999) An ICEy Death: Caspases are required for nuclear death in conjugating Tetrahymena thermophila. In: Senior Honor's Thesis. (Middletown: Wesleyan University)

39. Earnshaw WC (1995) Nuclear changes in apoptosis. Curr. Opin. Cell. Biol. 7 337-343

40. Lagarkova MA, larovaia OV and Razin S (1995) Large-scale fragmentation of mammalian DNA in the course of apoptosis proceeds via excision of chromosomal DNA loops and their oligomers. J. Biol. Chem. 270: 20239-2024

41. Zhivotovsky B, Wade D, Gahm A, Orrenius S and Nicotera P (1994) Formation of $50 \mathrm{kbp}$ chromatin fragments in isolated liver nuclei is mediated by protease and endonuclease activation. FEBS Lett. 351: 150 - 154

42. Nagata S (2000) Apoptotic DNA fragmentation. Exp. Cell Res. 256: 12-18

43. Allis $C D$ and Wiggins $J(1984)$ Histone rearrangements accompany nuclear differentiation and dedifferentiation in Tetrahymena. Dev. Biol. 101: 282-294

44. White EM, Allis CD, Goldfarb DS, Srivsatva A, Weir JW and Gorovsky MA (1989) Nucleus-specific and temporally restricted localization of proteins in Tetrahymena macronuclei and micronuclei. J. Cell Biol. 109: 1983-1992

45. Vaux D, Haecker G and Strasser A (1994) An evolutionary perspective on apoptosis. Cell 76: $777-779$

46. Cornillon S, Foa C, Davoust J, Buonavista N, Gross J and Golstein P (1994) Programmed cell death in Dictyostelium. J. Cell Sci. 107: 2691-2704

47. Kirk D (1994) Germ cell specification in Volvox carteri. Ciba Found. Symp. 182 2-15

48. Moreira M, Del Portillo H, Milder R, Balanco J and Barcinski M (1996) Heat shock induction of apoptosis in promastigotes of the unicellular organism Leishmania (Leishmania) amazonensis. J. Cell Physiol. 167: 305-313

49. Christensen S, Chemnitz J, Straarup E, Kristiansen K, Wheatley D and Rasmussen L (1998) Staurosporine-induced cell death in Tetrahymena thermophila has mixed characteristics of both apoptotic and autophagic degeneration. Cell Biol. Int. 22: 591-598

50. Wellburn S and Murphy N (1998) Prohibitin and RACK homologues are upregulated in trypanosomes induced to undergo apoptosis and in naturally occurring terminally differentiated forms. Cell Death Differ. 5: 615-622

51. Ameisen J (1998) The evolutionary origin and role of programmed cell death in single-celled organisms: A new view of executioners, mitochondria, hostpathogen interactions, and the role of death in the process of natural selection. In: When Cells Die, Lockshi T, ZakeriZ, Tilly J, eds (New York: Wiley Liss) pp. 3-56

52. Gömöri G (1941) Distribution of acid phosphatase in tissues under normal and under pathologic conditions. Arch. Path. 32: 189

53. Anderson O (1992) Cytochemical localization of acid phosphatase (lysosomal and digestive activity marker enzyme). In: Protocols in Protozoology, Lee J, Soldo A, eds (Lawrence, Kansas: Society of Protozoologists) pp. C22.1 - C22.7

54. Giloh H and Sedat JW (1982) Fluorescence microscopy: Reduced photobleaching of rhodamine and fluorescent protein conjugates by $n$ propylgallate. Science 271: 1252-1255 